\title{
LA FUERZA DE UN SIGNO. PERSPECTIVAS TEÓRICAS PARA EL ANÁLISIS DE LOS HASHTAGS \#
}

\section{THE STRENGTH OF A SIGN. THEORETICAL PERSPECTIVES FOR THE ANALYSIS OF HASHTAGS \#}

\author{
Gevisa La Rocca \\ Università degli Studi di Enna "Kore”, Enna / Italia \\ gevisa.larocca@unikore.it \\ https://orcid.org/0000-0003-2548-5473
}

Recibido/Received: 30/04/2020

Modificado/Modified: 06/06/2020

Aceptado/Accepted: 15/06/2020

\section{RESUMEN}

Los hashtags representan una forma extremadamente simple, utilizada por las redes sociales, para conectar noticias, temas, personas, emociones, eventos. Si en el primer período fue posible considerarlos exclusivamente como agregadores temáticos, ahora es necesario preguntarnos en qué se han convertido. $\mathrm{Al}$ analizar diferentes perspectivas teóricas, este artículo tiene como objetivo llegar a una reformulación de la definición del término y del concepto hashtag.

PALABRAS CLAVE

Acto lingüístico; creación de conocimiento; hashtag; ofrecimientos; simulacro.

\section{SUMARIO}

1. Introducción. 2. Buscando una Weltanschauung. 2.1 Marco teórico. 2.1.1 Perspectivas teóricas de Bernard. 2.1.2 Perspectivas teóricas de La Rocca. 3. Análisis de las diferentes perspectivas teóricas. 4. Conclusiones. Bibliografía.

\begin{abstract}
Hashtags represent an extremely simple way, used within the social network, to connect news, topics, people, emotions, events. If in the first period - it was possible - to consider them exclusively as thematic aggregators now it is necessary to ask ourselves what they have become. By analyzing different theoretical perspectives, this article aims to arrive at a definition of the term and concept of hashtag.
\end{abstract}

\section{KEYWORDS}

Speech acts; Hashtag; Knowledge creation; Affordances; Simulacrum.

\section{CONTENTS}

1. Introduction. 2. Looking for a Weltanschauung. 2.1 Theoretical frameworks. 2.1.1 Bernard's theoretical perspectives. 2.1.2 La Rocca's theoretical perspectives. 3. Analysis of the different theoretical perspectives. 4. Conclusions. References. 


\section{INTRODUCCIÓN}

Un hashtag es una etiqueta que representa un tema sobre el cual los usuarios de una red social pueden incluir una aportación u opinión personal con solo escribir la cadena de caracteres tras la almohadilla que da nombre a ese tema-etiqueta. Además, el hashtag constituye un mecanismo para indexar y recuperar mensajes relacionados con el tema correspondiente. Este trabajo pretende, de hecho, comprender e interpretar el uso que se viene haciendo de los hashtags. ¿Acaso son sólo herramientas para indexar temas de conversación, simples comentarios a los sucesos cotidianos? Sin duda, cuando aparecieron, su función era indexar conversaciones, pero no podemos ignorar que hoy son herramientas multitarea.

La popularidad de los hashtags está vinculada a su introducción en la red social Twitter, donde se utilizan como palabras clave que atraen la atención de los usuarios sobre un tema y actúan como recolectores de información y emociones. El diseño de este modo de comunicación se atribuye a Chris Messina, un abogado de San Francisco, quien lo difundió, aunque fue Nate Ritter el que le dio la popularidad a través de su perfil. Ritter, en octubre de 2007, incluyó \#sandiegofire en mensajes que hablaban sobre los incendios que afectaban al Condado de San Diego (Zak, 2013).

El uso masivo de hashtags tuvo lugar después de que Twitter lo introdujera en 2009 como una herramienta para agrupar mensajes (tweets), identificando en el hashtag una forma sencilla de registrar los temas. Un año después, Twitter introdujo en su primera página una cuestión que ha llegado a ser la más popular entre los usuarios de la red social dado que indica en qué medida los temas logran cierto nivel de impacto. A estos temas se les conoce como trending topics (temas de tendencia en un determinado momento), es decir, la lista de los hashtags más utilizados. En el año 2012 se introdujeron los temas de tendencias localizadas, que permiten mostrar los hashtags más populares para cada país; un año más tarde, Facebook también incorporó hashtags. En 2013, la American Dialect Society eligió hashtag para ser la palabra del año, y en 2014 fue aceptado en el Oxford English Dictionary (Bernard, 2019). Lexi Pandell (2017) advierte que inicialmente la palabra hashtag se escribió por separado, "hash tag". La propuesta para unificarlo fue de un informático, Stowe Boyd, quien estaba familiarizado con el símbolo \#, que generalmente se llamaba "hash" en su línea de trabajo, del lenguaje de programación $\mathrm{C}$.

Ya sea un evento, una protesta, la vida de un personaje famoso, el hashtag está compuesto por un significante \# y un significado, configurándose como un campo semántico compuesto de signos lingüísticos e imágenes mentales, continuamente redefinido a través de las acciones e interacciones humanas que le dan sentido (La Rocca, 2020). Por tanto, en términos lingüísticos, el hashtag existe en el umbral entre texto y metatexto y dibuja las fases previamente ocultas de catalogación e indexación (Bernard, 2019).

Bajo el concepto hashtag - o sea, un signo de almohadilla (hash \#) seguido de una etiqueta (tag) - se recopilan múltiples matices semánticos, y por ello es un paraguas bajo el cual se encuentran imágenes ocultas, significados, emociones; palabras que contribuyen, en fin, a expandir o modificar el significado original.

Elanor Colleoni (2013) sostiene que los hashtags son un significante con una amplia orientación polisémica, mientras que Zizi Papacharissi (2016) los define como significantes vacíos, abiertos a una operación de redefinición continua. También está el componente emocional que los usuarios asignan a los eventos, es decir, la formación del sentido de afecto público, de las audiencias conectadas (Boyd, 2010) que expresan su participación a través de expresiones de sentimiento (Papacharissi, 2016), que se juntan alrededor del propio 
hashtag. Por lo tanto, el hashtag va más allá de la etiqueta en sí y se abre a posibles mundos de significación que necesitan una especificación, porque solo si entendemos qué es cada hashtag, podremos identificar sus efectos en la construcción de la realidad social. De hecho, como sostiene Andreas Bernard (2019), en un mundo lleno de cosas materiales, el hashtag, en cambio, representa un compromiso: el compromiso de ver, de encontrar una audiencia y establecer un grupo de interés. Entonces, el \# ya no es un signo puramente funcional, porque esconde un compromiso social, siendo un símbolo social. Representa la producción y acumulación de atención pública. Por eso, es necesario leer los hashtag a través de lentes epistemológicas, lingüísticas, culturales y de medios de comunicación. En este trabajo, la atención se centra en el hashtag, qué es y qué impacto produce en la construcción de la realidad social, qué marcos teóricos se han utilizado, hasta la fecha, para explorar estos nuevos signos.

\section{BUSCANDO UNA WELTANSCHAUUNG}

Si el objetivo al que se dedican los científicos sociales que estudian los hashtags es la comprensión, entendida como Verstehen (Abel, 1948), comprender su historia, la matriz teórica debe ser uno de los principales pasos a dar, también para elegir, más adelante, el enfoque metodológico más correcto para su análisis. Si no está claro qué son los hashtags, ¿cómo se pueden estudiar correctamente?

En Theory of Hashtag, Bernard (2019) sigue una línea de análisis retrospectiva para definir la importancia de este signo. Se dedica a la biografía de un símbolo, indicando la historia del mismo en el campo de la impresión, luego explica el uso del signo \# en los teléfonos y encuentra elementos de conexión con las palabras clave. Bernard no descuida informar la importancia de este símbolo en estudios recientes, relacionándolo con actividades tan distantes como el activismo político y el marketing. Igual que hacen Gevisa La Rocca (2020) o La Rocca y Cirus Rinaldi (2020), Bernard se pregunta cómo este signo ha cambiado la organización y distribución lingüística, cultural, mediática de declaraciones y documentos. El objetivo declarado de este trabajo es conectar y analizar estas diferentes perspectivas teóricas y, de esta manera, definir los hashtags. Para lograr este objetivo, revisamos las principales perspectivas teóricas que han surgido en los últimos años relacionadas con los hashtags.

\subsection{Marco teórico}

En este apartado se presentan las perspectivas teóricas utilizadas por Bernard y La Rocca para comprender cómo entender el uso y la esencia de un hashtag.

\subsubsection{Perspectivas teóricas de Bernard}

El punto de partida del trabajo de Bernard es la búsqueda de los tipos de cambio que ha implicado la introducción de este signo, entendido como una lengua franca. Para hacer esto, desarrolla tres caminos: a) el estado fisionómico del hashtag; b) la arqueología del saber de Michel Foucault (1972) y c) la organización linguiística, mediática y social vinculada a este símbolo.

a) El estado fisionómico del hashtag

Bernard acude a Theodor Adorno (1990), quien define el estado fisionómico de la puntuación como su capacidad de constituir su propia expresión, que no puede separarse de 
la función sintáctica que desempeñan, pero al mismo tiempo no termina con ella. Entonces hay algo más: en la apertura de su artículo, Adorno describe los signos de puntuación como una transcripción de los elementos de la comunicación no verbal; recordando cómo el signo de exclamación se asemeja a un dedo levantado, el punto y coma a un bigote. Después de todo, nada más cierto, si piensas en el uso que en la comunicación mediada por computadora de los primeros años se les hizo para dibujar caras, que se convirtieron en nuestros emoticones y luego en emojis. Adorno continúa su discusión describiendo el uso de la puntuación en la música, en la construcción de partituras, en la asignación de un tempo y un ritmo, y luego se centra en la escritura de novelistas. En este último caso, especifica cómo hay reglas de puntuación que los escritores deben respetar, y cómo el uso que hacen de ellas las distingue y, sobre todo, cómo los escritores no pueden, por un lado, confiar ciegamente en las reglas, que a menudo son rígidas y ásperas, y, por otro, ignorar las reglas por completo. No pueden ignorarlos por completo porque a través de la puntuación pueden llamar la atención del lector sobre lo que es fundamental en su narración. La puntuación otorga énfasis o atenúa las palabras. En la última parte de su artículo, Adorno aclara perfectamente la fisonomía de los signos de puntuación, destacando cómo, en cada acto de puntuación, se puede decir si hay una intención o no en su uso. Para decirlo más sutilmente, uno puede percibir la diferencia entre una voluntad subjetiva (la del escritor) que demuele brutalmente las reglas de puntuación socialmente aceptadas, o una sensibilidad táctil (típica de los escritores) que permite que las reglas resuenen en el fondo, incluso donde están suspendidos. Por lo tanto, los signos de puntuación, su selección, las formas en que se utilizan construyen un significado que se convierte en sentido colectivo. Este enfoque es el que está detrás de la siguiente afirmación de Bernard:

A good 60 years later, it is time to consider the "physiognomic status" of the hashtag in today's digital culture, and particularly to consider the extent to which this symbol has influenced such things as the use of language or the creation of collectives (Bernard, 2019: 132).

\section{b) La arqueología del saber}

Con la intención de describir e interpretar los hashtags, Bernard recurre a la obra de Foucault Arqueología del saber (1972). Lo hace porque dentro de este trabajo metodológico, el único escrito por Foucault, hay margen de maniobra para comenzar a definir cómo, en realidad, a partir de la idea del agregador temático, el hashtag oculta una red de significados mucho más complejos. Esta red es evidente cuando se piensa en cómo los diferentes significados de un hashtag pueden interactuar, mezclarse, dentro de la red social. De hecho, en el trabajo de Foucault se presenta una "ley de distribución", que regula lo que puede y no puede decirse dentro de un campo temático específico y en un lugar y tiempo asimismo específicos. La misma ley implica la idea de que diferentes declaraciones se entrelazan, generando manifestaciones colectivas de interés y creación de significado. Tomemos como referencia la parte del trabajo de Foucault que se dedica a las formaciones discursivas, lo que lleva a preguntarnos qué es lo que realmente estamos diciendo cuando hablamos de gramática, medicina, economía política. En una primera hipótesis, se puede argumentar que diferentes enunciados forman un corpus teórico definido, ya que se refieren al mismo objeto. Sin embargo, Foucault nos dice que esta creencia de que se refieren al mismo objeto es solo una ilusión pura, ya que cada objeto del que hablamos se forma de manera diferente de acuerdo con las declaraciones que emiten quienes lo nombran, explican y delimitan. En esta perspectiva foucaultiana, la cuestión a abordar cambia porque ya no se trata de buscar la unicidad y la persistencia de un objeto, sino de descubrir, identificar el espacio en el que se 
perfilan y transforman continuamente los diferentes objetos.

Una característica distintiva de Foucault es la identificación. Esta no ocurre en la persistencia de los temas, sino en la descripción de los momentos de ruptura, de dispersión, de las correlaciones entre las declaraciones, al encontrar su espacio común, la conexión en sus transformaciones. En el caso de que se pueda describir un sistema de dispersión de este tipo, identificando sus regularidades, o más bien un orden en las transformaciones, correlaciones, operaciones, nos enfrentaremos a una formación discursiva. Las condiciones de existencia, a las que responden los elementos de esta red, representan las reglas de entrenamiento que caracterizan una división discursiva específica.

En este punto, es necesario completar estas reglas de capacitación con contenidos. Foucault habla primero de todos los conceptos que cambian según las edades; luego de "instancias de delimitación", refiriéndose a los diversos campos del conocimiento que se identifican de manera diferente $\mathrm{y}$, al hacerlo, delimitan objetos; finalmente, establece el concepto de "cuadrículas de especificación", esto es, "contenedores" a los que nos referimos al hablar de cierto objeto. Pero el discurso no coincide solo con el lugar donde los objetos previamente estructurados se superponen y se encuentran, ya que el objeto no espera en un lugar apartado e inmóvil, sino que existe en las condiciones positivas de un complejo rango de relaciones, las cuales no determinan el objeto en su textura interna, sino que son lo que permite que el objeto aparezca de cierta manera.

Las relaciones discursivas, por lo tanto, no son algo interno para el discurso o las jerarquías, pero tampoco son exclusivamente externas. En cambio, caracterizan el discurso como una práctica, luego especifican el conjunto de relaciones que el discurso debe llevar a cabo para hablar sobre estos y esos objetos, y al mismo tiempo proporcionan las herramientas para poder realizar operaciones fundamentales, tales como analizar, clasificar, o explicar, entre otras. Esto no significa mirar más allá del discurso, convirtiéndolo en el signo de otra cosa, sino más bien hacerlo emerger en toda su rica complejidad. Los discursos no deben considerarse como un conjunto de signos que se refieren a contenidos o representaciones ya dados, sino como prácticas que forman los mismos objetos de los que hablan.

El enfoque de Foucault se define, por lo tanto, por su carácter relacional. Como él mismo dice, no hay una declaración en general, como tampoco hay una declaración libre, neutral e independiente. De hecho, una declaración siempre pertenece a una serie o un todo, y siempre juega un papel fundamental entre otras afirmaciones. A partir de aquí, Bernard desarrolla su razonamiento sobre los hashtags, preguntándose cuáles pueden ser las implicaciones del símbolo \# con respecto a la agrupación y subsunción del conocimiento. La almohadilla es precisamente lo que favorece al hashtag como organizador de sentimientos, opiniones y expresiones (su capacidad para ser identificado y clasificado, su concentración en ciertos temas) y lo que establece sus límites. Bernard se inspira en los conceptos de Foucault, quien escribe que una afirmación siempre pertenece a una serie o a un todo y que es necesario establecer sus correspondencias con otras afirmaciones para comprenderla, y sostiene que lo mismo debe hacerse con los hashtags. Bernard va más allá y afirma que, hoy, el trabajo intelectual del historiador ha sido facilitado por los hashtags, por su función de organización automática de los contenidos. En el pasado, para obtener una nueva categoría analítica como la "declaración", era necesario modificar el archivo y sus categorías tradicionales de "autor" y "trabajo". Sin embrago, dentro de una red social como Twitter o Instagram, un solo clic es todo lo que se necesita para organizar las publicaciones por palabra clave. Por lo tanto, al hacer explícito el método de conexión en la red social, el hashtag facilita ver las relaciones entre las declaraciones, los “sistemas de dispersión”, como los llamó Foucault. Además, la 
palabra clave con el signo hash (almohadilla) confirma la definición central de "afirmación" de Foucault:

In The Archaeology of Knowledge, he repeatedly remarks that, for historical analysis, it is not the semantic content of this unit that is of primary interest but rather its "function"; as he envisioned it, discourse analysis is not a "semantic investigation" (Bernard, 2019: 249).

Por esta razón, Bernard dice que la inclusión del hashtag en los mensajes de Twitter se puede describir exactamente de la misma manera:

its power derives from the distribution of statements and documents in a strictly functional sense, one that is independent of the meaning of the tweets or photographs in question. A post may be relevant, competent, poignant, or factually correct, but this plays no role whatsoever in its ability to be networked by means of a hashtag (Bernard, 2019: 249).

Entonces subraya cómo es:

The software tool makes decisions according to its program and not by conducting hermeneutic assessments on an individual basis. Previously fleeting categories such as "attention" or "resonance" have thereby become quantifiable in the countable accumulation of posts and in the "trending" of particular keywords (Bernard, 2019: 249).

a) La organización lingüística, mediática y social

Está claro que para Bernard el hashtag no es solo un signo, sino un símbolo que construye una red llena de significados y es capaz de activar conexiones colectivas. La idea de que el hashtag debe estudiarse como algo más que el signo utilizado para crearlo parte de esta suposición. Bernard cita el estudio de Paola Caleffi (2015), donde los hashtags se examinan como elementos lingüísticos producidos a través de un mecanismo potencialmente nuevo de formación de palabras. Se trata del mecanismo de hashtagging, que se agregaría a los procesos morfológicos existentes. Específicamente, Caleffi confirma que el hashtag es ciertamente una palabra nueva por derecho propio, pero también sugiere que el hashtag puede considerarse el producto de un nuevo proceso morfológico, por lo tanto, como un mecanismo que puede generar una variedad casi ilimitada de formas al agrupar palabras, generando por ende nuevos elementos lingüísticos. Estos son elementos se definen como palabras y como no palabras. Pero, también, es posible definirlos como "hiperpalabras" (palabras aumentadas), que transforman "el mundo en un hipertexto, es decir, le da un nuevo significado" (Niola, 2014: 9), de tal modo que pueden servir para actuar como una referencia a un argumento o para crear comunidades de personas interesadas en un tema. De hecho, los hashtags promueven la afiliación con los valores y las comunidades que los apoyan, haciendo posible que los usuarios de Twitter interactúen con otros y, por lo tanto, amplifiquen sus métodos de comunicación (Dickinson, 2013). En este punto, Bernard se pregunta si anteriormente había algo así:

Where was the keyword before the hashtag? What function did it serve in the formation of the public sphere and in the arrangement of archives? Who created it, and which operations were responsible for its dissemination? (Bernard, 2019: 430431).

Sin embargo, desde el comienzo de su historia, las palabras clave no han desempeñado una de las funciones principales de los hashtags: capturar emociones. En efecto, la función de las palabras clave era y es indexar, identificar y organizar el contenido. Así, es posible afirmar que, durante el siglo XX, los hashtags tienen dos precedentes: palabras clave como 
unidad de organización de documentos y palabras clave como temas, temas más debatidos en una era histórica o dentro de una agenda política. Así es que el hashtag se usa para indexar y organizar la información, pero al mismo tiempo se convierte en un eslogan y a través de su análisis (lingüístico, semántico) se recupera información sobre "cómo" hablamos "de qué". Por su capacidad de ser conectores polisémicos, se convierten en una herramienta útil en campañas políticas o en acciones políticas dirigidas al activismo, siendo asimismo un instrumento del marketing propiamente dicho. De hecho, ya en 2011, Axel Bruns y Jean Burgess publicaron un artículo teórico sobre el uso de hashtags en Twitter, reflexionando sobre la creación de resistencia política a través de ellos. De este modo, destacaron el papel central del hashtag en la coordinación de audiencias conectadas en la red social. Asimismo, en el trabajo de Nathan Rambukkana (2015), se recopilan numerosos estudios de comunicación y activismo político a través de hashtags. El estudio de Yarimar Bonilla y Jonathan Rosa (2015) analiza la acción llevada a cabo por el hashtag \#ferguson en la protesta callejera en Missouri y también documenta la fuerza de lo que se ha llamado hashtag activism. El mismo estudio muestra cómo las plataformas de redes sociales se han convertido en sitios poderosos para documentar y desafiar episodios de brutalidad policial y declaraciones falsas contenidas en los principales medios de comunicación. De tal modo, el activismo creado con el uso de hashtags puede forjar una acción política compartida para desafiar y reescribir los motivos de una protesta. Esta fortaleza del hashtag no ha podido escapar del marketing (Nichols, 2017), pues - a través de él - expande las capacidades virales de las campañas publicitarias (Bennett, 2014; Chen, 2018). Por lo tanto, también en el análisis de Bernard hay una indicación de cómo esta herramienta emerge de la red social y contribuye a rediseñar una nueva realidad.

\subsubsection{Perspectivas teóricas de La Rocca}

Para analizar los hashtags, La Rocca utiliza las contribuciones de: a) John Searle y la teoría de los actos lingüísticos; b) las affordances (los ofrecimientos, concebido como conjunto de estímulos mediante los que un objeto proporciona a un organismo (agente) la oportunidad de realizar una acción); c) el hashtag como producto cultural.

a) Searle y la teoría de los actos lingüísticos

La pregunta inicial de La Rocca (2020) es: ¿cómo se pueden analizar correctamente los hashtags si uno no sabe cuáles son? Por lo tanto, su interés está dirigido a identificar una metodología de investigación con hashtags, a partir de su definición. Buscando un marco teórico para los hashtags, La Rocca elige la teoría de los actos lingüísticos de Searle (1969) porque sirve para conectar el significado de un hashtag (pensado como una palabra escrita) con fotos, imágenes, emojis y enlaces que se adjuntan a esa etiqueta textual. La Rocca presume, así pues, que el significado de un hashtag depende de todo lo que los usuarios le adjunten o asocien. Por ello considera particularmente apropiado el análisis de contenido multimodal (La Rocca, 2018a y 2018b), puesto que el contenido de un hashtag está construido a partir de un conjunto de elementos, de modo que, si se persigue estudiar el significado de los hashtags, es preciso analizar todos los elementos vinculados, en congruencia con aportaciones recientes procedentes del big data y otros modelos matemáticos:

In fact, according to Bruns et al. 2016 the hashtag studies can be understood as representing the dominant stream of Twitter, but they are a "low hanging fruit in social media data (p. 1) (La Rocca, 2020: 3). 
Para comprender los motivos de la asimilación de los hashtags a la categoría de actos ilocucionarios, es necesario seguir los argumentos desarrollados por Searle en Speech Acts (1969) y en The Construction of Social Reality (1995). La Rocca desarrolla este tema en algunos artículos, pero principalmente en el de 2020. Seguimos su camino aquí. Comienza analizando el pensamiento de Searle sobre el lenguaje. En efecto, Searle (1969) concibe el lenguaje como una forma de comportamiento, por lo tanto, cree que hablar un idioma equivale a involucrarse en una forma de comportamiento, de hecho, hablar es para él realizar actos lingüísticos. Los actos lingüísticos que distingue son cuatro: el acto de expresión, el acto proposicional (compuesto por la referencia y la predicación), el acto ilocutivo y el acto perlocutor. De acuerdo con las interpretaciones dadas por Harold Innis (1951), según las cuales el propio carácter de la comunicación depende del medio que lo transmite, se puede argumentar que hoy los hashtags están dando lugar a una nueva forma de interpretar los actos lingüísticos, con consecuencias que se remontan a la construcción de la realidad social, entendida como una relación constante entre la mente individual y la sociedad que contribuye a la creación del mundo social (Searle, 2010).

Searle (1969), en sus obras, se centra en los actos ilocutivos y perlocutivos,

Considering however the illocution as the 'complete' speech act, or rather as the speech act par excellence. Pronouncing an expression in a grammatically correct way, but without a sense or a reference, is not communicating. On the other hand, saying something - with both a sense and reference - but without a reason for who is listening, is not communicating. The distinction between illocutions and perlocutions is different. The latter refer to the effects of communication, and go beyond communication itself (La Rocca y Rinaldi, 2020: 31).

En esencia, para Searle, la ilocución es el acto lingüístico por excelencia y sirve como paradigma para la referencia y la predicación, es decir, para los dos actos que juntos constituyen el acto proposicional. Según Searle, al manifestar una indignación se dice algo, se transmite un contenido y se provoca un efecto en los interlocutores, a modo de consecuencia. De hecho, esta diferencia de nivel se ha codificado en el lenguaje: al emitir una declaración en la que se expresa una indignación, la parte expresiva no supone necesariamente una declaración completa, mientras que una oración solo puede ser una parte de la expresión misma. En relación con los efectos, Searle plantea que pueden no ser lingüísticos. Al establecer este paralelismo entre la ilocución y los hashtags, y, por lo tanto, al considerar los hashtags como actos lingüísticos, La Rocca considera fundamental la distinción de Searle entre el sentido, el significado y la referencia, que para él no son equivalentes, sino dos momentos del acto proposicional (La Rocca, 2020; La Rocca y Rinaldi, 2020).

Partiendo de la presunción de que significar algo y decir algo significativo son aspectos de la declaración, como actividades tanto intencionales como convencionales, es fácil deducir que el hashtag se asimila al acto ilocutivo. Un hashtag es una afirmación acompañada por una proposición u otra afirmación, pero también un contenido multimodal, representado por imágenes, videos, enlaces, emojis que se adjuntan a él (La Rocca, 2018a).

Según Searle, no es suficiente con estudiar los usos del lenguaje (Smith, 2003), porque otros problemas filosóficos permanecen abiertos. Uno principalísimo se refiere a la naturaleza de la obligación o del poder, así como la noción de responsabilidad. Estas conectan con una cuestión más amplia: la libertad, voluntariedad o racionalidad de los actos comunicativos personales. Para resolver este enigma es necesario estudiar no sólo el lenguaje, sino también el cerebro, la mente, las leyes de la física, y las formas de organización social. Así es como después de una serie de contribuciones sobre la filosofía 
del lenguaje y la expansión de la nueva teoría del acto lingüístico, Searle se aventura en un nuevo territorio, con trabajos sobre la intencionalidad, la mente y la conciencia. Aquí se sigue el mismo camino. Para comprender qué se entiende por intencionalidad, relacionándola con los actos lingüísticos, con la mente o con el estado de las cosas, es necesario distinguir los fenómenos que son relativos al observador de los fenómenos que resultan independientes del observador en abstracto, considerando cómo la explicación de dos puntos de vista diferentes contribuye al desarrollo de dos perspectivas de análisis.

La Rocca sigue el razonamiento de Searle, y por eso se pregunta:

If we return to the hashtags we use on Twitter, what intrinsically contains a

hashtag, considered separately from observers, users or other forms of external

intentionality? (La Rocca y Rinaldi, 2020: 32).

Dicho lo cual, pensando en Twitter, la pregunta obvia que se plantea es: ¿qué contiene un hashtag de manera intrínseca, per se, independientemente de los observadores, usuarios u otras formas de intencionalidad externa? La respuesta es simple: intrínsecamente contiene caracteres digitales compuestos de bits que pasan de un estado a otro y lo hacen muy rápidamente. El quid de la pregunta es el siguiente: en el tránsito de un estado a otro, somos nosotros los que escribimos, tuiteamos y retuiteamos los hashtags para realizar ciertas funciones, pero estas funciones son siempre relativas al observador. Se puede argumentar que el hashtag contiene información en sí mismo, sólo porque el observador sabe cómo interpretar y a qué se refiere el hashtag. Por lo tanto, hay un sentido de intencionalidad que es independiente del observador (intencionalidad intrínseca), un sentido de intencionalidad que es extrínseco al observador (intencionalidad extrínseca), y luego hay una tercera forma de atribución de intencionalidad, que es metafórica. Esta distinción hecha por Searle es sin duda relevante al extrapolarlo al significado de los hashtags y su remodulación en los diferentes usos que exhiben los miembros de las redes sociales. Por lo tanto, es necesario distinguir entre los usos literales y el carácter intencional de los hashtag. Concretamente, deben ser diferenciados a) los usos literales que describen estados intrínsecos o independientes del observador; y b) los usos literales que describen estados intencionales que son tales solo en relación con un observador (La Rocca, 2018a; La Rocca y Rinaldi, 2020). Estas dos aplicaciones literales de nociones intencionales se distinguen a su vez de las aplicaciones metafóricas de las nociones intencionales. La aclaración de Searle (2010) es fundamental, ya que sostiene que no es posible tener un estado intencional sin tener muchos otros en los que se presuponen creencias, valores, deseos y pueden considerarse como una red, en la que funciona todo estado intencional. Es decir, determina las propias condiciones de satisfacción, solo para su posición y su relación con todos los demás en la red. Toda la red de intencionalidad funciona sólo porque hay un fondo, un campo que hace posible que los elementos de detalle de la red funcionen correctamente.

Such background does not consist of further beliefs added to the web itself, but rather of a habitus, shared social practices, ways of being correlated to it in some way (La Rocca y Rinaldi, 2000: 32).

Por estas razones, La Rocca puede argumentar que el fondo no consiste en creencias adicionales que se agregan a la red en sí misma, sino que es un habitus (Bourdieu, 1972), prácticas sociales compartidas, formas de relacionarse con él de alguna manera. Debido a ello el hashtag se convierte en un paraguas semántico, un conector polisémico, un coleccionista de emociones siempre relacionadas con él pero que lo amplifican y redibujan, reelaborándose los matices originales entre significado y sentido. 
b) Los ofrecimientos

La Rocca usa los ofrecimientos para explicar cómo cambia el significado de los hashtags a través de la interacción humana. El ofrecimiento es un concepto propio de la teoría ecológica de la percepción (Gibson, 1979), que posteriormente se adoptó en otros campos. Estas son las propiedades del entorno circundante que actúan de estímulos que permiten u ofrecen el potencial de acción de un agente. Como muchos estudios han demostrado (Wells, 2002), los ofrecimientos no son simplemente propiedades del entorno/ambiente: existen solo como una relación complementaria entre un agente y su entorno circundante. Taina Bucher y Anne Helmond (2018) hicieron una revisión de los usos del concepto de ofrecimiento, e informan que este concepto ha sido examinado desde diferentes perspectivas: ofrecimiento de alto y bajo nivel (boyd, 2010), ofrecimientos imaginarios (Nagy y Neff, 2015) y ofrecimientos vernáculos (Mcveigh-Schultz y Baym, 2015). Además, Bucher y Helmond (2018) en su análisis de las plataformas sociales muestran cómo estos pueden permitir diferentes acciones para varios tipos de usuarios (incluidos los usuarios finales $\mathrm{o}$ desarrolladores), o cambios en la plataforma. Para Chamil Rathnayake y Daniel Suthers (2018), los hashtags de Twitter pueden verse como ofrecimientos por dos razones: 1) la plataforma ofrece la creación de hashtags y 2) a través de los hashtags surgen diferentes tipos de acción. La Rocca agrega una tercera: 3) la posibilidad de que los hashtags cambien su significado original a través de la operación de retuitear o citar.

El estudio de Rathnayake y Suthers (2018) se basa en un análisis de interacción independiente en los medios:

... so is not subject to the constraints that offline metaphors carry over to the

analysis of onlinetransactions, and therefore provides a foundation for a natively

digital conception of phenomenological elements of online expressions (Rathnayake

y Suthers 2018: 2).

Partiendo del concepto de uptake (recepción) definido como "acts in which one participant takes up another's contribution and does something further with it" (Suthers, 2006: 331), añaden el concepto de momentary connectedness, o "conectividad momentánea", la cual se define como:

... a novel conception of online publicness, as an extended computer-mediated sociality that includes transactive as well as non-transactive online activity (Rathnayake y Suthers, 2018: 2).

Relacionado con los anteriores, proponen un tercer concepto, el de "projected uptake" (recepción proyectada), que consistiría en el "most fundamental element of interaction" (Suthers et al. 2010), basado en el ofrecimiento de actos para futuras recepciones (Rathnayake y Suthers, 2018). El objetivo con el que introducen estos dos conceptos es examinar "transactive as well as non-transactive elements in Twitter hashtags" (Rathnayake y Suthers, 2018). Porque para ellos, los hashtags son ofrecimientos de la plataforma que organizan instancias de conexión momentánea en redes. La Rocca utiliza estos estudios para demostrar que el hashtag cambia a través de la interacción humana, modificando así su significado original. Esta observación se vuelve central cuando se trabaja empíricamente con hashtags, porque siempre debemos tener en cuenta que el hashtag tiene un significado denotativo y connotativo, uno visible y otro latente, uno que viene de arriba en un proceso deductivo y otro emergiendo desde abajo en un proceso inductivo.

c) El hashtag como producto cultural

Si se considera que el hashtag es un producto cultural, que cambia su significado a través 
de las acciones e interacciones humanas, es necesaria la búsqueda de un marco teórico que satisfaga tal consideración. Al indagar este marco teórico debemos tener en cuenta dos advertencias: la primera, cobrar conciencia de que estamos inmersos en el momento presente. La segunda - bien subrayada por la sociología del conocimiento -, recordar que la "forma" de las herramientas de comunicación solo puede entenderse después de que la próxima tecnología se haya manifestado. Por lo tanto, la radio se puede entender después del advenimiento de la televisión, la televisión después de la computadora, la computadora después de Internet, Internet después de las redes sociales. De este entrelazamiento de conocimiento hay "objeciones culturales", un fragmento de una totalidad cognitiva más amplia. Estas son formaciones de significado, que se utilizan en el acto cognitivo y que de alguna manera deberían devolvernos, a través de su entrelazamiento, la totalidad del fenómeno (Mannheim, 1952).

En efecto, Mannheim aborda el problema metodológico del estudio científico de los objetos culturales, siguiendo un enfoque fenomenológico y considerándolos a la par con las "unidades sensoriales estructuradas". Los objetos culturales se colocan en una especie de esfera o nivel intermedio entre la comprensión teórica racional y la irracionalidad, razón por la cual el estudio de estos objetos requiere una interpretación de capas de significado: a) el sentido objetivo; b) el sentido intencional de la expresión; c) el sentido documental (1952).

¿De qué se trata? A diferencia de un objeto natural, para comprender el objeto o producto cultural, es necesario no solo penetrar en su sentido objetivo, sino que también debe considerarse como una expresión y un documento que nos informa sobre algo, si la voluntad es comprender completamente su naturaleza. La Rocca (2020) ofrece el hashtag \#MeToo como ejemplo, que también fue usado de manera independiente y casi simultánea por Bernard (2019). Así se expresa La Rocca sobre este hashtag, convertido en fenómeno social:

For example, the hashtag \#MeToo had a literal meaning when it was put on the social networks, and it was launched by a feminist movement against violence on women. However, it became viral in October 2017, as a hashtag used by social media to help show the widespread presence of sexual violence and harassment against women after the public revelation of the accusations of sexual violence against Harvey Weinstein. If we now carry out a research on such hashtag, we can understand how its original meaning has been widened, transfigured, through human interactions, that is through its usage and spreadability (Jenkins, 2006) (La Rocca, 2020: 5).

Es claro que el hashtag \#MeToo da cuerpo a un problema social, ubicado en un momento histórico, económico y político dado, del que los usuarios de las redes sociales se han convertido en voceros y difusores.

Se deduce que las formas de objetos culturales que asume la vida diaria deben considerarse formaciones de significado. El significado también se encuentra en la expresión, o sentido expresivo. Este no es separable del sujeto y de su experiencia vivida; de hecho, la expresión del significado adquiere su comprensión y singularidad solo si se coloca en relación con el mundo interior de quienes lo expresan. La expresión del mundo interior a través del objeto cultural vuelve a quienes se colocan en interacción con él mediante el sentido documental. En otras palabras, es una traducción del significado del objeto o de la acción que restaura el habitus de quienes lo realizan. Es por estas razones que el sentido documental es entendible solo para el observador. Para comprender este mecanismo es necesario dividir el proceso de apropiación del significado en dos etapas: a) el contenido 
representado y su forma de representación; b) el aspecto del contenido del medio de representación, por lo tanto, el material utilizado y la forma que asume (el medio). Tomemos \#MeToo como referencia, si nos limitamos al contenido representado, inmediatamente encontramos el elemento objetivo y el elemento expresivo: forma y contenido. Por un lado, el movimiento feminista en cuanto tal, y, por otro, sus demandas y funciones educativa, informativa y divulgativa que genera el contenido de las publicaciones de los usuarios en torno a este movimiento. Notamos de inmediato que la distinción entre forma y contenido es fluida. Otra distinción que se puede hacer es respecto al sentido documental, es decir, el valor que el observador atribuye a este hashtag o nube de hashtags.

¿Qué función desempeña el hashtag \#MeToo para quienes lo generaron y para quienes lo retuitearon? Para visualizar la distinción, es preciso pensar en dos públicos: simpatizantes y detractores. Está claro que diferentes momentos expresivos se atribuyen a los mismos eventos y a las mismas figuras y que esto depende del usuario. Sin embargo, para tratar de comprender la intención del creador del hashtag e interpretar correctamente la capa de significado, uno debe haber estudiado en profundidad las intenciones sensoriales de ese momento y del creador específico (Mannheim, 1952). La consecuencia de esto es que los objetos culturales de la vida cotidiana (y los hashtags lo son) deben considerarse formaciones sensoriales. Por supuesto, trazar el origen del sentido significa entrar en el mundo del significado. Esto requiere entrar en el espacio reflexivo de la intencionalidad y en una dimensión simbólica compuesta de experiencias colectivas y del intercambio social de símbolos. La creación de sentido surge de la intersección del área subjetiva pre-reflexiva con el área reflexiva y transitiva del intercambio social (Weick, 1995). Por tanto, cuando analizamos los datos extraídos de las redes sociales e indexados mediante el uso de hashtags, debemos tener en cuenta la importancia de la construcción social del hashtag y los significados que le subyacen. Lo mismo ha de hacerse si los usamos para el análisis de audiencias (Athique, 2018) que para el de eventos catastróficos (Matheson, 2018). En los dos casos, los hashtag nacen con una atribución de sentido particular, pero que cambia a través de la interacción, desarrollando y enriqueciendo los "posibles seres" de cada hashtag.

Para resumir el proceso interpretativo incrustado en los hashtags, La Rocca (2020) utiliza el modelo teórico de Wendy Griswold (1997). Si nos dejamos guiar por su esquema de "diamantes culturales", tenemos: a) un productor crea un \#hashtag y lo coloca en el mundo social representado aquí por las redes sociales, b) es reutilizado por los receptores, usuarios de las redes sociales que lo reinterpretan y, mediante su trabajo de citas o retwitts c) lo transforman en un objeto cultural a través de medios comunicativos. Los receptores que transforman el hashtag en un objeto cultural permiten que se manifieste los "possible selves" ("seres posibles") del hashtag (La Rocca, 2020). Por tal concepto se quiere indicar:

... we mean to say that a hashtag can have multiple expressions has a multiplicity of expressions it can give a form to. In other words, it can become something more than what it contains, and this self-pertinent form is potentially inserted in it. Possible selves of a hashtag are trusted to the agency of users, who allow them to emerge, contributing to the construction of social reality (Searle, 1995) and producing an impact on the social world (Griswold, 1997). By having both, a signifier and a meaning, a hashtag can change the content while moving from one tweet to another, thanks to the dynamic character of the concept it contains, through interaction, knowledge, and motivation (La Rocca, 2020: 2).

A lo largo de este proceso es posible conocer porciones de significado. El sentido objetivo está representado por la fuente o por el productor; el sentido expresivo se usa para 
rastrear y encerrar la intención del producto cultural y está encerrado en la acción del receptor, el sentido documental devuelve información sobre en qué se ha convertido el hashtag en el pasaje de un tweet a otro, es decir, el viaje que hace como producto cultural.

\section{ANÁLISIS DE LAS DIFERENTES PERSPECTIVAS TEÓRICAS}

Está claro que un hashtag es más que el signo que lo representa. En este punto, es necesario preguntarse si existe, en este momento, una posible definición de en qué se ha convertido este signo. Las perspectivas teóricas presentadas aquí, aunque formuladas por dos autores, recuerdan numerosas contribuciones de otros académicos. La gama de posibilidades interpretativas es, por lo tanto, amplia. Entonces tenemos una primera necesidad, que es organizar y agrupar estas líneas de interpretación. Con el objetivo de construir un esquema de clasificación, tomamos prestados otros conceptos de otros autores. Específicamente, usamos la idea de un simulacro desarrollado por Jean Baudrillard (1978; 2010) y, posteriormente, le injertamos un aparato empírico, representado por el análisis de objetos culturales realizado por Karl Mannheim (1952).

¿Por qué podría el concepto de simulacro ser bueno para definir hashtags? Para Baudrillard, el simulacro es un fenómeno que atraviesa las sociedades contemporáneas y que se caracteriza por el surgimiento de la hiperrealidad, la simulación, la confusión entre signo y sentido. La misma confusión que se ha asumido aquí ocurre con los hashtags. Además, encontramos la hiperrealidad como fenómeno definitorio del tiempo, que surge precisamente de esta actitud, que no es de disimulo, sino de simulación, la cual lleva a la anulación del objeto por su reflejo. Llegamos a las mismas conclusiones cuando argumentamos que hay más en el hashtag que el signo que lo representa. El objetivo es reconstruir la realidad de los hashtags, con las mismas intenciones que Baudrillard tiene (1978: 23), cuando dice "constituye el simulacro total que recupera la 'realidad' mediante una circunvolución completa", y todavía "su red de signos artificiales se enlazará inextricablemente con elementos reales" (1978: 44).

Para analizar el hashtag como un simulacro, consideramos el trabajo desarrollado por Baudrillard para la ficción (2010), donde el autor identifica tres órdenes de simulacros: 1) los simulacros naturales, o naturalistas, basado en la imagen, imitación, falsificación; 2) los simulacros productivos, o productivistas, basados en la energía, fuerza; 3 ) los simulacros de simulación, basados en la información, el modelo, la hiperrealidad. Partiendo de esta triple distinción, es preciso ver si estos elementos se encuentran también dentro del hashtag y de las líneas interpretativas que se han proporcionado. Alcanzar la meta, en algún momento, casi parecerá "que sin haberlo querido se encontrará usted inmerso de lleno en lo real — una de cuyas funciones es precisamente la de devorar toda tentativa de simulación, la de reducir todas las cosas a la realidad" (Baudrillard, 1978: 44). Para no perderse exclusivamente en un mundo hiperreal, es necesario leer los simulacros uniéndolos a algo operacionalizable: el sentido objetivo, el sentido subjetivo, el sentido documental. De esta forma, se crea una tabla de doble entrada, capaz de darnos - como porcentaje total de la fila en estadísticas - un resultado. La suma conceptual de los tres resultados puede ser útil para encontrar una definición de hashtags, según expone la tabla 1. 
Tabla 1 - Resumen de las perspectivas teóricas y los significados de un hashtag

\begin{tabular}{|c|c|c|c|}
\hline \multicolumn{4}{|c|}{ Simulacro natural } \\
\hline & Bernard & La Rocca & Resultado \\
\hline $\begin{array}{l}\text { Sentido } \\
\text { objetivo }\end{array}$ & $\begin{array}{l}\text { Signo de } \\
\text { puntuación, } \\
\text { lenguaje de } \\
\text { programación } \\
\text { de } \\
\text { ordenadores, } \\
\text { palabras clave }\end{array}$ & $\begin{array}{l}\text { Acto lingüístico que lo } \\
\text { determina como } \\
\text { compuesto de una } \\
\text { proposición más otras } \\
\text { modalidades }\end{array}$ & $\begin{array}{l}\text { El hashtag tiene sus propias } \\
\text { características que se derivan de } \\
\text { ser un signo gráfico, derivado de } \\
\text { la programación de } \\
\text { computadoras, con valor } \\
\text { linguístico }\end{array}$ \\
\hline \multicolumn{4}{|c|}{ Simulacro productivo } \\
\hline & Bernard & La Rocca & Resultado \\
\hline $\begin{array}{l}\text { Sentido } \\
\text { subjetivo }\end{array}$ & $\begin{array}{l}\text { Construcción } \\
\text { de un } \\
\text { significado } \\
\text { reticular }\end{array}$ & $\begin{array}{l}\text { Ofrecimientos } \\
\text { producidos por la } \\
\text { interacción humana }\end{array}$ & $\begin{array}{l}\text { El significado de un hashtag no } \\
\text { es inmutable con el tiempo. } \\
\text { Depende de cómo lo usen los } \\
\text { actores sociales y de cómo se } \\
\text { desarrolle una red de significados } \\
\text { conectados conectado con ello }\end{array}$ \\
\hline \multicolumn{4}{|c|}{ Simulacro de simulación } \\
\hline & Bernard & La Rocca & Resultado \\
\hline $\begin{array}{l}\text { Sentido } \\
\text { documental }\end{array}$ & $\begin{array}{l}\text { Activismo } \\
\text { político, } \\
\text { campañas de } \\
\text { marketing }\end{array}$ & $\begin{array}{l}\text { Los posibles seres se } \\
\text { convierten en la } \\
\text { manifestación tangible } \\
\text { de los cambios de } \\
\text { hashtag }\end{array}$ & $\begin{array}{l}\text { El uso voluntario o involuntario } \\
\text { de un hashtag causa efectos, } \\
\text { determinando su multiplicidad de } \\
\text { significados y rangos de acción. } \\
\text { Son estos elementos los que se } \\
\text { convierten en objeto de estudio } \\
\text { de los académicos, a partir de } \\
\text { ellos es posible reconstruir tanto } \\
\text { los significados que se han } \\
\text { atribuido a un hashtag en un } \\
\text { momento dado, como también los } \\
\text { efectos producidos en los medios } \\
\text { y en la realidad social }\end{array}$ \\
\hline
\end{tabular}

Fuente: Elaboración propia a partir del texto presentado aquí

\section{CONCLUSIONES}

El Oxford English Dictionary, en junio de 2014, agregó el nombre del hashtag entre sus entradas, definiéndolo de esta manera: a word or phrase with the symbol '\#' in front of it, 
used on social media websites and apps so that you can search for all messages with the same subject; deteniéndose en un nivel puramente descriptivo del signo gráfico y sus funciones.

El trabajo expuesto en este texto sugiere, sin embrago, que el hashtag es un fenómeno extremadamente complejo, que no recogen adecuadamente los diccionarios. El hashtag es un signo gráfico derivado del lenguaje de programación de computadoras que adquiere un valor lingüístico. Asume un valor lingüístico porque el signo gráfico que se utiliza para indicarlo no se puede separar del texto que se adjunta al signo. El hashtag, que consiste en un significado y un significante, se convierte en una etiqueta con un valor comunicativo tanto a nivel descriptivo como connotativo. Por lo tanto, puede realizar dos macrofunciones: agregador semántico y agregador colectivo. Además, el significado de un hashtag no es inmutable, sino que varía, y ello depende de haber admitido que tiene un significado connotativo, que los actores redefinen continuamente al utilizarlo, ya sea en la red social, en los principales medios de comunicación o en la realidad cotidiana. El significado de un hashtag está, por tanto, sujeto a continuas redefiniciones hechas por usuarios sociales, lo que implica asimismo que cada hashtag tiene múltiples seres, múltiples identidades significativas. De esta multiplicidad, los estudios de hashtag solo captan (o producen) el sentido documental que en un tiempo y espacio social determinado le han atribuido los actores-agentes.

\section{BIBLIOGRAFÍA}

Abel, T. (1948). "The Operation Called Verstehen". American Journal of Sociology, 54(5): 211-218.

Adorno, T.W. (1990). "Punctuation Marks". The Antioch Review, 48(3): 300-305.

Athique, A. (2018). "The dynamics and potential of big data". Media, Culture \& Society, 40(1): 59-74.

Baudrillard, J. (1978). Cultura y simulacro. Barcelona: Editorial Kairós.

Baudrillard, J. (2010). Cyberfilosofia. Milano: Mimesis Edizioni.

Bennett, S. (2014). "The History of the Hashtag in Social Media Marketing". Adweek, www.adweek.com/digital/history-hashtag-social-marketing

Bernard, A. (2019). Theory of Hashtag. Cambridge: Polity Press (Amazon Kindle).

Bonilla, Y., Rosa J. (2015). "\#Ferguson: Digital protest, hashtag ethnography, and the racial politics of social media in the United States". American Ethnologist, 00(0): pp. 4-16, https://doi.org/10.1111/amet.12112

Bourdieu, P. (1972). Esquisse d'une théorie de la pratique précedé de Trois études d'ethnologie kabile. Paris: Seul.

Boyd, d. (2010). "Social Network Sites as Networked Publics: Affordances, Dynamics, and Implications". En Z. Papacharissi (Ed.) A Networked Self: Identity, Community, and Culture on Social Network Sites. New York: Routledge, pp. 39-58.

Bruns, A. y Burgess, J. (2015). "Twitter hashtags from ad hoc to calculated publics". En N. Rambukkana (Ed.) Hashtag Publics: The Power and Politics of Discursive Networks. New York: Peter Lang, pp 13-28.

Bruns, A.; Moon, B.; Paul, A. y Muench, F.V. (2016). “Towards a typology of hashtag publics: a large scale comparative study of user engagement across trending topics. Communication Research and Practice, 2(1): 20-46, DOI: 10.1080/22041451.2016.1155328

Bucher, T., y Helmond, A. (2018). "The affordances of social media platforms”. En J. Burgess, A. Marwick, y T. Poell (Eds.) The SAGE Handbook of Social Media. London: Sage, pp. 233-253.

Caleffi, P.M. (2015). “The 'Hashtag': A New Word or a New Rule?" SKASE Journal of Theoretical Linguistics, 12: 46-69, en http://www.skase.sk/Volumes/JTL28/pdf doc/05.pdf

Chen, J. (2018). "How to Use Hashtag Marketing to Dramatically Boost Brand Awareness". Sprout Social, https://sproutsocial.com/ 
Colleoni, E. (2013). "Beyond the Differences: The Use of Empty Signifiers as Organizing Device in the \#occupy Movement". Workshop Material Participation: Technology, the Environment and Everyday Publics, Milan: University of Milan.

Dickinson, P. (2013). " $\mathrm{B} / \mathrm{w}$ U \& me': The Functions of Formulaic Language in Interactional Discourse on Twitter". The Linguistic Journal, 7(1): 7-38.

Foucault, M. (1972). The Archaeology of Knowledge \& The Discourse on Language. New York: Pantheon Books.

Gibson, J.J. (1979). "The theory of affordances". En J. J. Gibson (Ed.) The ecological approach to visual perception. classic edition. Boston: Psychology Press, pp. 119-136.

Griswold, W. (1997). Cultures and Societies in a Changing World. London: Sage.

Innis, H.A. (1951). The Bias of Communication. Toronto: The University of Toronto Press.

Jenkins, H. (2006). Convergence Culture: Where Old and New Media Collide. New York: New York University Press.

La Rocca, G. (2018a). "Against Big Data? Using Multimodal Content Analysis to reconstruct a semantic of hashtags". International Conference on Unspoken, Unseen, Unheard of Unexplored realities in qualitative research, St. Gallen, Switzerland.

La Rocca, G. (2018b). Nuove forme di comunicazione sociale. Antifragilità, communication voice e studio di caso. Roma: Carocci.

La Rocca, G. (2020). "Possible selves of a hashtag: Moving from the theory of speech acts to cultural objects to interpret hashtags". International Journal of Sociology and Anthropology, 12(1): 1-9, DOI: $10.5897 / \mathrm{IJSA} 2019.0833$

La Rocca, G. y Rinaldi, C. (2020). "LGBTQI+ icons between resistance and normalization: looking for mediatization of emotions in hashtags". International Review of Sociology/Revue Internationale de Sociologie, 30(1): 26-45, https://doi.org/10.1080/03906701.2020.1724367

Mannheim, K. (1952). Essays on the Sociology of Knowledge. London: Routledge and Kegan Paul.

Matheson, D. (2018). "The performance of publicness in social media: tracing patterns in tweets after a disaster". Media, Culture and Society, 40(4): 584-599, https://doi.org/10.1177/0163443717741356

Mcveigh-Schultz, J. y Baym, N.K. (2015). "Thinking of you: Vernacular affordance in the context of the microsocial relationship app Couple". Social Media + Society, 1(2).

Messina, C. (2007). "Groups for Twitter; or Proposal for Twitter Tag Channels". Factory Joe, https://factoryjoe.com/2007/08/25/groups-for-twitter-or-a-proposal-for-twitter-tagchannels

Nagy, P. y Neff, G. (2015). "Imagined affordance: Reconstructing a keyword for communication theory". Social Media+Society, 1(2).

Nichols, P. (2017). "Hashtag Marketing: How to Use Hashtags that Fit Your Brand". Blue Fountain Media, www.bluefountainmedia.com/blog/hashtag-marketing-for-your-brand

Niola, M. (2014). HASHTAG. Cronache di un paese connesso. Milano: Bompiani.

Pandell, L. (2017). "An Oral History of the \#Hashtag". Wired (May 19, 2017), www.wired.com/2017/05/oral-history-hashtag

Papacharissi, Z. (2015). "Affective publics and structures of storytelling: Sentiment, events and mediality”. Information, Communication \& Society, 19: 307-324, DOI: 10.1111/j.14602466.2012.01630.x

Rambukkana, N. (Eds.) (2015). Hashtag Publics. The Power and Politics of Discursive Networks. New York: Peter Lang.

Rathnayake, C. y Suthers, D.D. (2018). "Twitter Issue Response Hashtags as Affordances for Momentary Connectedness" Social Media+ Society, July-September, 1-14, https://doi.org/10.1177/2056305118784780

Searle, J. R. (1969). Speech Acts. An Essay in the Philosophy of Language. Cambridge: Cambridge University Press.

Searle, J. R. (1995). The Construction of Social Reality. New York: The Free Press.

Searle, J. R. (2010). Making the Social World. The Structure of Human Civilization. Oxford: Oxford University Press.

Smith, B. (2003). "John Searle: From speech acts to social reality". En Smith, B. (Ed.) John Searle. Cambridge: Cambridge University Press, pp. 1-33. 
Suthers, D. D. (2006). "Technology affordances for intersubjective meaning making: A research agenda for CSCL". International Journal of Computer-Supported Collaborative Learning, 1(3): 315-337.

Suthers, D. D., Dwyer N., Medina, R., y Vatrapu, R. (2010). "A framework for conceptualizing, representing, and analyzing distributed interaction". International Journal of Computer-Supported Collaborative Learning, 5(1): 5-42.

Weick, K. E. (1995). Sensemaking in Organizations. London: Sage.

Wells, A. J. (2002). "Gibson's affordances and Turing's theory of computation". Ecological Psychology, 14(3): 140-180.

Zak, E. (2013). "How Twitter's Hashtag Came to Be". The Wall Street Journal, 3 octubre 2013, en https://blogs.wsj.com/digits/2013/10/03/how-twitters-hashtag-came-to-be/

\section{Breve currículo:}

\section{Gevisa La Rocca}

Profesora titular de sociología de la comunicación en la Universidad Kore de Enna. Es coordinadora científica del grupo de investigación internacional Rischio, comunicazione e società/ Riesgo, comunicación y sociedad, con este equipo publicó estos volúmenes como coeditora: En torno al Riesgo. Contribuciones de diferentes disciplinas y perspectivas de análisis (Pasos Edita, 2018) y Technological and Digital Risk: Research Issues (Peter Lang, 2020). Sus intereses de investigación incluyen la mediatización de emociones, riesgos y entornos digitales, comunicación social, estudios de hashtag, análisis de datos cualitativos. Sobre estos temas ha publicado artículos y libros. Actualmente está trabajando con un equipo en el hashtag \#coronavirus, para comprender las reacciones de los italianos en Twitter a la triple crisis producida por la pandemia. 